\title{
All-Cause Mortality And Cardiovascular Events In A Spanish Nonagenarian Cohort According To Type 2 Diabetes Mellitus Status And Established Cardiovascular Disease
}

\author{
MA Salinero-Fort ( $\square$ miguel.salinero@salud.madrid.org) \\ Subdirección General de Investigación y Documentación. Consejería de Sanidad. Calle Aduana \\ J Mostaza-Prieto \\ Hospital Carlos III, IdIPAZ, Madrid \\ C Lahoz-Rallo \\ Hospital Carlos III, IdIPAZ, Madrid \\ J Cárdenas-Valladolid \\ Universidad Alfonso X El Sabio, Instituto de Investigación Sanitaria del Hospital Universitario La Paz (IdiPAZ) \\ JI Vicente-Díez \\ Centro Salud Monóvar, FIIBAP, Madrid \\ P Gómez-Campelo \\ Instituto de Investigación Sanitaria del Hospital Universitario La Paz (IdiPAZ) \\ JM de Miguel-Yanes \\ Hospital General Universitario Gregorio Marañón, Universidad Complutense de Madrid (UCM), Instituto de Investigación \\ Sanitaria Gregorio Marañón (IiSGM)
}

\section{Research Article}

Keywords: Nonagenarians, diabetes mellitus, cardiovascular disease, mortality rates

Posted Date: May 28th, 2021

DOI: https://doi.org/10.21203/rs.3.rs-457930/v1

License: (9) (i) This work is licensed under a Creative Commons Attribution 4.0 International License. Read Full License

Version of Record: A version of this preprint was published at BMC Geriatrics on March 18th, 2022. See the published version at https://doi.org/10.1186/s12877-022-02893-z. 


\section{Abstract}

Background: To analyze all-cause mortality and cardiovascular events in nonagenarians from a Mediterranean population.

Methods: Primary Health Care (265 health centres)

Participants: Primary health records of all the nonagenarians living in the Community of Madrid $(N=59,423)$. We defined 4 groups according to prevalent type 2 diabetes mellitus and established cardiovascular disease: (1) type 2 diabetes mellitus $(-)$, cardiovascular disease (-); (2) type 2 diabetes mellitus (-), cardiovascular disease (+); (3) type 2 diabetes mellitus (+), cardiovascular disease (-); and (4) type 2 diabetes mellitus (+), cardiovascular disease (+). Follow-up time was 2.5 years.

Primary outcome: Cumulative incidence and the density incidence rates of all-cause mortality, non-fatal myocardial infarction, non-fatal stroke (combined they constituted the first composite primary outcome (CPO1)), and heart failure (similarly, combined with the previous they constituted CPO2). We evaluated the adjusted effect of groups classification on all-cause mortality (Cox regression).

Results. Mean age was $93.3 \pm 2.8$ years; $74.2 \%$ were women. Hypertension, dyslipidemia, heart failure, albuminuria and estimated glomerular filtration rate $<60 \mathrm{~mL} / \mathrm{min} / 1.73 \mathrm{~m}^{2}$ were significantly more prevalent in group 4 (type 2 diabetes mellitus $(+)$, cardiovascular disease $(+)$ ) than in the other groups (all p values $<0.001$ ). We observed significantly higher rates of cumulative incidence of all-cause mortality, CPO1 and CPO2 in participants belonging to group 4 (all $p$ values $\leq 0.001$ ). People in group 2 (type 2 diabetes mellitus (-), cardiovascular disease (+)) presented higher rates of all-cause mortality, heart failure, CPO1 and CPO2 than people in group 3 (type 2 diabetes mellitus (+), cardiovascular disease (-)) (all $p$ values $\leq 0.001$ ). In the fully-adjusted model, prevalent type 2 diabetes mellitus plus established cardiovascular disease (group 4) independently predicted all-cause mortality $(\mathrm{HR}=1.48(95 \% \mathrm{Cl}, 1.40$ to 1.57$)$ as compared to reference group 1 (type 2 diabetes mellitus $(-)$, cardiovascular disease $(-))(p<0.01)$. Also, cardiovascular disease and type 2 diabetes mellitus alone showed significant HRs $(1.13$ and 1.14 , respectively; both $p$ values $<0.01)$ ).

Conclusions. In Spanish nonagenarians, established cardiovascular disease and type 2 diabetes mellitus conferred modest all-cause mortality risks. The concurrent presence of both conditions conferred the highest all-cause mortality risk.

\section{Background}

It is widely accepted that type 2 diabetes mellitus (T2DM) [1, 2] and established cardiovascular disease (CVD) [3, 4] increase morbidity and mortality risks. The burden of T2DM and established CVD to influence incident cardiovascular events and mortality may differ according to gender, ethnic background, environmental conditions or the age of the population. The Hoorn study, in the Dutch population aged 50 to 75, showed that women with T2DM and no history of CVD had a cardiovascular risk similar to women without T2DM and history of CVD, whereas in men the presence of established CVD conferred the highest cardiovascular risk [5]. In a recent study also coming from Spain, illustrative of a Mediterranean country, people with T2DM had more than double the risk of ischemic stroke after adjusting for other risk factors, whilst T2DM appeared as an independent factor for in-hospital mortality only in women [6].

More specifically, few studies have focused on the natural history of cardiovascular disease in the very old population, since old frail people are usually excluded from randomized controlled trials [7, 8]. As a consequence, in the American Diabetes Association's 2021 review of "Standards of Medical Care in Diabetes", the chapter dedicated to diabetes treatment in older adults cites no single randomized controlled trial evaluating the impact of hypoglycemic treatment on cardiovascular morbimortality in the elderly [9]. One rare exception to the rule is the HYVET study, which highlighted the beneficial impact of hypertension treatment on incident cardiovascular disease in the senior community [10].

\section{Methods}


Here, we aimed to compare the incidence of all-cause mortality and cardiovascular events among four subsets of the Spanish nonagenarian population: (1) individuals without T2DM or established CVD (T2DM (-), CVD (-)), (2) individuals without T2DM but with established CVD (T2DM (-), CVD (+)), (3) individuals with T2DM but without established CVD (T2DM $(+)$, CVD (-)), and (4) individuals with both T2DM and established CVD (T2DM (+), CVD (+)), taking into account the influence of the gender of the participants on the outcomes.

\section{Study design}

The study design has been previously published [11]. Briefly, we performed a population-based study in two phases: first, a cross-sectional study of all residents aged $\geq 90$ years living in the Community of Madrid (Spain); second, a 2.5-year follow-up study of this population. The Community of Madrid is a public entity providing healthcare coverage to $100 \%$ of the population. It provides primary care through 3,881 general practitioners working in 265 health centres. All residents have an electronic clinical record in primary care. The record constitutes a clinical database of anonymised data. As at 31 December 2015 , it contained data for 61,059 persons aged $\geq 90$ years. Data were available for 59,423 subjects, of whom 11,645 (19.6\%) had been diagnosed with T2DM.

\section{Variables and definitions}

Data for all participants $(n=59,423)$ were collected through electronic clinical records in primary care, and included age, sex, cardiovascular risk factors, comorbidities and medication prescriptions as at 31 December 2015. Comorbidities were recorded according to the International Classification of Primary Care-Second Edition (ICPC-2).

Basic and instrumental activities of daily life were measured using the Barthel Index [12] and the Lawton and Brody Scale [13], respectively. As potential confounders, we collected information on the items included in the Charlson's comorbidity index [14], which predicts 10-year mortality according to a wide range of comorbid conditions (i.e., age, dementia, chronic obstructive pulmonary disease (COPD), diabetes with or without organ damage, connective tissue disease, chronic kidney disease (CKD), AIDS, liver disease, malignancy, congestive heart failure, established acute myocardial infarction, peripheral vascular disease, transient ischemic attack or stroke, hemiplegia, and peptic ulcer disease). Dementia was identified by the P70 code of ICPC-2.

CVD was defined as a diagnosis of angina pectoris, myocardial infarction, stroke or peripheral artery disease in the electronic clinical record (ICPC-2 codes K74, K75, K76, K90 and K92). The mortality data including date of death was extracted from the SIP-CIBELES database (Community Information System of the Community of Madrid), which has records for the entire population of individuals in the Community of Madrid who hold a public health card (with the right to access public health care services). Only all-cause mortality was available with no information on the cause of death.

\section{Follow-up}

All participants were followed-up during 30 months (2.5 years), a time period that was deemed sufficient for the development of events given the age of the population. We calculated the cumulative incidence and the density incidence rates dividing the number of incident cases by the number of person-years of follow-up $(x 1,000)$ for the following variables: all-cause mortality, non-fatal myocardial infarction, non-fatal stroke, the first composite primary outcome (CPO1) -defined as all-cause mortality, non-fatal myocardial infarction and non-fatal stroke-, and the second composite primary outcome (CPO2) -defined as all-cause mortality, non-fatal myocardial infarction, non-fatal stroke and heart failure-, for both men and women with and without T2DM, stratified by the absence or presence of established CVD.

The quality of the primary care electronic clinical record has previously been validated for research purposes [15], and the database has been widely used to study the epidemiology of cardiovascular risk factors in the study population [16]. Statistical analysis

Continuous variables are presented as mean and standard deviation (SD), and categorical variables as percentages. Categorical variables were compared using the chi-square test. Continuous variables were compared using the t-test. 
Analysis of variance (ANOVA) was conducted with the Dunnett's T3 or Scheffe's post hoc comparison test, and was used to compare the prespecified groups according to prevalent T2DM and established CVD, respectively.

The cumulative incidence values are expressed as either a rate (events/person-years) or as a proportion (events/person). The confidence interval $(\mathrm{Cl})$ at $95 \%$ of the crude incidence rates were calculated using the exact Poisson formula. The adjusted effect of the groups on all-cause mortality was analyzed by the Cox regression model, and the corresponding hazard ratios (HRs), and their 95\% Cls were calculated. The adjusting variables were: age, sex, chronic obstructive pulmonary disease, solid cancer, leukemia/lymphoma, chronic kidney disease, dementia, heart failure, deep vein thrombosis/pulmonary thromboembolism, and atrial fibrillation (these clinical diagnoses are part of the Charlson's comorbidity index). Data were processed using SPSS for Windows, V.19.0 (IBM Corp., Armonk, New York, USA).

\section{Results}

At baseline, the cohort consisted of 59,423 nonagenarians, with a mean age of $93.3 \pm 2.8$ years, of whom $74.2 \%$ were women. Group 1 (T2DM (-), CVD (-)) was comprised by 37,078 people (77.3\% women), group 2 (T2DM (-), CVD (+)) by 10,700 people (64.4\% women), group 3 (T2DM (+), CVD (-)) by 8,043 people (76.4\% women), and group 4 (T2DM (+), CVD (+)) by 3,602 people (65.6\% women) (Table 1 and Supplemental Table S1). In each group, women were older, more frequently lived in nursing homes, had lower rates of functional independence, higher blood pressure and LDL-cholesterol levels, higher prevalence of obesity, hypertension, dyslipidemia and impaired renal function, but lower smoking rates than men (Supplemental Table S1).

Hypertension, dyslipidemia, heart failure, albuminuria and estimated glomerular filtration rate (eGFR) $<60 \mathrm{~mL} / \mathrm{min} / 1.73 \mathrm{~m}^{2}$ were significantly more prevalent in group $4($ T2DM $(+)$, CVD $(+))$ than in the other groups (all p values $<0.001)$. In addition, people in group 4 significantly more often received treatment with antiplatelet agents, anticoagulants, statins and angiotensin-converting enzyme inhibitors or angiotensin receptor blockers than people in the other groups (all $p$ values $<0.001$ ) (Table 1). People in group $3($ T2DM (+), CVD (-)) had a higher prevalence of hypertension, dyslipidemia and albuminuria, and a lower prevalence of heart failure and atrial fibrillation than people in group 2 (T2DM (-), CVD (+)) (all p values $<0.001)$ (Table 1). 
Table 1

Baseline characteristics of the population of nonagenarians included in the study, according to type 2 diabetes mellitus (T2DM) status, and the presence or absence of prior cardiovascular disease (CVD).

\begin{tabular}{|c|c|c|c|c|c|c|c|}
\hline & $\begin{array}{l}\text { Group 1: } \\
\text { No T2DM, no } \\
\text { prior CVD } \\
(n=37,078)\end{array}$ & $\begin{array}{l}\text { Group 2: } \\
\text { No T2DM, } \\
\text { prior CVD } \\
(n=10,700)\end{array}$ & $\begin{array}{l}\text { Group 3: } \\
\text { T2DM, no } \\
\text { prior CVD } \\
(n=8,043)\end{array}$ & $\begin{array}{l}\text { Group 4: } \\
\text { T2DM, prior } \\
\text { CVD } \\
(n=3,602)\end{array}$ & $\mathrm{p}_{\text {value }}$ & $\begin{array}{l}\text { p value (for } \\
\text { comparison } \\
\text { between } \\
\text { group 2 } \\
\text { and group } \\
3 \text { ) }\end{array}$ & $\begin{array}{l}\text { p value (for } \\
\text { comparison } \\
\text { between } \\
\text { group } 3 \\
\text { and group } \\
\text { 4) }\end{array}$ \\
\hline $\begin{array}{l}\text { Age (years), } \\
\text { mean (SD) }\end{array}$ & $93.3(2.8)$ & $93.3(2.7)$ & $92.9(2.5)$ & $92.9(2.4)$ & $\dot{0} 001$ & $<0.001$ & 0.581 \\
\hline $\begin{array}{l}\text { Living in } \\
\text { nursing home, } \\
\text { n/N (\%) }\end{array}$ & $\begin{array}{l}5,479 / 37,078 \\
(14.8)\end{array}$ & $\begin{array}{l}1,342 / 10,700 \\
(12.5)\end{array}$ & $\begin{array}{l}1,098 / 8,043 \\
(13.7)\end{array}$ & $\begin{array}{l}489 / 3,602 \\
(13.6)\end{array}$ & <. 001 & 0.015 & 0.884 \\
\hline $\begin{array}{l}\text { Barthel: } \\
\text { Functionally } \\
\text { independent, } \\
\mathrm{n} / \mathrm{N}(\%)\end{array}$ & $\begin{array}{l}1,591 / 13,779 \\
(12.5)\end{array}$ & $\begin{array}{l}451 / 4,865 \\
(9.3)\end{array}$ & $\begin{array}{l}411 / 3,747 \\
(11.0)\end{array}$ & $\begin{array}{l}151 / 1,884 \\
(8.0)\end{array}$ & $\dot{0} 001$ & 0.009 & $<0.001$ \\
\hline $\begin{array}{l}\text { Charlson's } \\
\text { index, mean } \\
\text { (SD) }\end{array}$ & $4.8(1.1)$ & $5.9(1.3)$ & $6.0(1.2)$ & $7.1(1.4)$ & $<.001$ & $<0.001$ & $<0.001$ \\
\hline $\begin{array}{l}\mathrm{SBP}(\mathrm{mmHg}) \\
\text { mean }(\mathrm{SD})\end{array}$ & $130.3(16.7)$ & $128.6(16.9)$ & $\begin{array}{l}131.5 \\
(16.5)\end{array}$ & $\begin{array}{l}130.2 \\
(18.1)\end{array}$ & $\dot{0} 001$ & $<0.001$ & 0.015 \\
\hline $\begin{array}{l}\mathrm{DBP}(\mathrm{mmHg}) \text {, } \\
\text { mean }(\mathrm{SD})\end{array}$ & 70.7 (9.4) & $69.7(9.7)$ & $70.5(9.4)$ & $69.2(9.7)$ & <. 001 & $<0.001$ & $<0.001$ \\
\hline $\begin{array}{l}\mathrm{BMI}>30 \\
\mathrm{~kg} / \mathrm{m}^{2}, \mathrm{n} / \mathrm{N}(\%)\end{array}$ & $\begin{array}{l}2,597 / 13,048 \\
(19.9)\end{array}$ & $\begin{array}{l}815 / 4,140 \\
(19.7)\end{array}$ & $\begin{array}{l}907 / 3,764 \\
(24.1)\end{array}$ & $\begin{array}{l}333 / 1,617 \\
(20.6)\end{array}$ & $<.001$ & $<0.001$ & 0.005 \\
\hline $\begin{array}{l}\text { Current smoker, } \\
\mathrm{n} / \mathrm{N}(\%)\end{array}$ & $\begin{array}{l}289 / 19,250 \\
(1.5)\end{array}$ & $\begin{array}{l}136 / 6,368 \\
(2.1)\end{array}$ & $\begin{array}{l}81 / 5,134 \\
(1.6)\end{array}$ & $\begin{array}{l}40 / 2,378 \\
(1.7)\end{array}$ & 0.001 & 0.048 & 0.750 \\
\hline $\begin{array}{l}\text { Hypertension, } \\
\mathrm{n} / \mathrm{N}(\%)\end{array}$ & $\begin{array}{l}25,010 / 37,078 \\
(67.5)\end{array}$ & $\begin{array}{l}7,936 / 10,700 \\
(74.2)\end{array}$ & $\begin{array}{l}6,505 / 8,043 \\
(80.9)\end{array}$ & $\begin{array}{l}2,978 / 3,602 \\
(82.7)\end{array}$ & $\begin{array}{l}<.001 \\
0.00\end{array}$ & $<0.001$ & 0.020 \\
\hline $\begin{array}{l}\text { Dyslipidemia, } \\
\mathrm{n} / \mathrm{N}(\%)\end{array}$ & $\begin{array}{l}11,903 / 37,078 \\
(32.1)\end{array}$ & $\begin{array}{l}4,452 / 10,700 \\
(41.6)\end{array}$ & $\begin{array}{l}3,640 / 8,043 \\
(45.3)\end{array}$ & $\begin{array}{l}1,860 / 3,602 \\
(51.6)\end{array}$ & $\begin{array}{l}< \\
0.001\end{array}$ & $<0.001$ & $<0.01$ \\
\hline $\begin{array}{l}\text { LDL- } \\
\text { Cholesterol } \\
\text { (mg/dl), mean } \\
\text { (SD) }\end{array}$ & $109.5(30.4)$ & $97.4(31.4)$ & $98.6(30.4)$ & $88.8(31.2)$ & $\begin{array}{l}< \\
0.001\end{array}$ & 0.155 & $<0.001$ \\
\hline $\begin{array}{l}\text { Chronic atrial } \\
\text { fibrillation, } \mathrm{n} / \mathrm{N} \\
\text { (\%) }\end{array}$ & $\begin{array}{l}5,322 / 37,078 \\
(14.4)\end{array}$ & $\begin{array}{l}2,485 / 10,700 \\
(23.2)\end{array}$ & $\begin{array}{l}1,424 / 8,043 \\
(17.7)\end{array}$ & $\begin{array}{l}846 / 3,602 \\
(23.5)\end{array}$ & $<.001$ & $<0.001$ & 0.041 \\
\hline $\begin{array}{l}\text { Heart failure, } \\
\mathrm{n} / \mathrm{N}(\%)\end{array}$ & $\begin{array}{l}3,355 / 37,078 \\
(9.0)\end{array}$ & $\begin{array}{l}1,573 / 10,700 \\
(14.7)\end{array}$ & $\begin{array}{l}1,040 / 8,043 \\
(12.9)\end{array}$ & $\begin{array}{l}666 / 3,602 \\
(18.5)\end{array}$ & $\begin{array}{l}< \\
0.001\end{array}$ & $<0.001$ & $<0.001$ \\
\hline $\begin{array}{l}\text { Albuminuria, } \\
\mathrm{n} / \mathrm{N}(\%)\end{array}$ & $\begin{array}{l}1,379 / 5,296 \\
(26.0)\end{array}$ & $\begin{array}{l}532 / 1,741 \\
(30.6)\end{array}$ & $\begin{array}{l}1,084 / 2,814 \\
(38.5)\end{array}$ & $\begin{array}{l}541 / 1,209 \\
(44.7)\end{array}$ & $\begin{array}{l}< \\
0.001\end{array}$ & $<0.001$ & $<0.001$ \\
\hline
\end{tabular}

SBP: Systolic blood pressure; DBP: Diastolic blood pressure; BMI: Body mass index; eGFR: estimated glomerular filtration rate; ACEl: Angiotensin-converting enzyme inhibitors; ARB: Angiotensin receptor blockers. 


\begin{tabular}{|c|c|c|c|c|c|c|c|}
\hline & $\begin{array}{l}\text { Group 1: } \\
\text { No T2DM, no } \\
\text { prior CVD } \\
(n=37,078)\end{array}$ & $\begin{array}{l}\text { Group 2: } \\
\text { No T2DM, } \\
\text { prior CVD } \\
(n=10,700)\end{array}$ & $\begin{array}{l}\text { Group 3: } \\
\text { T2DM, no } \\
\text { prior CVD } \\
(n=8,043)\end{array}$ & $\begin{array}{l}\text { Group 4: } \\
\text { T2DM, prior } \\
\text { CVD } \\
(n=3,602)\end{array}$ & $\begin{array}{l}\mathrm{p} \\
\text { value }\end{array}$ & $\begin{array}{l}\text { p value (for } \\
\text { comparison } \\
\text { between } \\
\text { group } 2 \\
\text { and group } \\
3 \text { ) }\end{array}$ & $\begin{array}{l}\text { p value (for } \\
\text { comparison } \\
\text { between } \\
\text { group } 3 \\
\text { and group } \\
\text { 4) }\end{array}$ \\
\hline $\begin{array}{l}\text { eGFR }<60 \\
\mathrm{~mL} / \mathrm{min} / 1.73 \\
\mathrm{~m}^{2}, \mathrm{n} / \mathrm{N}(\%)\end{array}$ & $\begin{array}{l}6,128 / 10,300 \\
(59.5)\end{array}$ & $\begin{array}{l}2,103 / 3,369 \\
(62.4)\end{array}$ & $\begin{array}{l}1,770 / 2,787 \\
(63.5)\end{array}$ & $\begin{array}{l}880 / 1,307 \\
(67.3)\end{array}$ & $<.001$ & 0.373 & 0.017 \\
\hline $\begin{array}{l}\text { Antiaggregants, } \\
\mathrm{n} / \mathrm{N}(\%)\end{array}$ & $\begin{array}{l}9,191 / 37,078 \\
(24.8)\end{array}$ & $\begin{array}{l}6,896 / 10,700 \\
(64.4)\end{array}$ & $\begin{array}{l}2,834 / 8,043 \\
(35.2)\end{array}$ & $\begin{array}{l}2,445 / 3,602 \\
(67.9)\end{array}$ & $\begin{array}{l}< \\
0.001\end{array}$ & $<0.001$ & $<0.001$ \\
\hline $\begin{array}{l}\text { Anticoagulants, } \\
\mathrm{n} / \mathrm{N}(\%)\end{array}$ & $\begin{array}{l}6,163 / 37,078 \\
(16.6)\end{array}$ & $\begin{array}{l}2,708 / 10,700 \\
(25.3)\end{array}$ & $\begin{array}{l}1,589 / 8,043 \\
(19.8)\end{array}$ & $\begin{array}{l}947 / 3,602 \\
(26.3)\end{array}$ & $<.001$ & $<0.001$ & $<0.001$ \\
\hline $\begin{array}{l}\text { ACEl or ARB, } \\
n / N(\%)\end{array}$ & $\begin{array}{l}11,940 / 37,078 \\
(32.2)\end{array}$ & $\begin{array}{l}4,530 / 10,700 \\
(42.3)\end{array}$ & $\begin{array}{l}3,371 / 8,043 \\
(41.9)\end{array}$ & $\begin{array}{l}1,764 / 3,602 \\
(49.0)\end{array}$ & $<.001$ & 0.580 & $<0.001$ \\
\hline Statins, n/N (\%) & $\begin{array}{l}7,093 / 37,078 \\
(19.1)\end{array}$ & $\begin{array}{l}4,895 / 10,700 \\
(45.7)\end{array}$ & $\begin{array}{l}2,795 / 8,043 \\
(34.8)\end{array}$ & $\begin{array}{l}1,981 / 3,602 \\
(55.0)\end{array}$ & $<.001$ & $<0.001$ & $<0.001$ \\
\hline
\end{tabular}

We observed significantly higher rates of cumulative incidence of all-cause mortality, CPO1 and CPO2 in participants belonging to group 4 (all p values $\leq 0.001$ ) (Table 2). People in group 2 presented higher rates of all-cause mortality, heart failure, CPO1 and CPO2 than people in group 3 (all p values $\leq 0.001$ ), but a lower rate of incident acute myocardial infarction $(p=0.015)$, with no difference in the rate of stroke $(p=0.547)$ (Table 2$)$. 
Table 2

Cumulative incidence of all-cause mortality and cardiovascular events in the nonagenarian population of Madrid after 2 years of follow-up, according to type 2 diabetes mellitus (T2DM) status, and the presence or absence of prior cardiovascular disease (CVD).

\begin{tabular}{|c|c|c|c|c|c|c|c|}
\hline & $\begin{array}{l}\text { Group 1: } \\
\text { No T2DM, } \\
\text { no prior } \\
\text { CVD } \\
(n= \\
33,091)\end{array}$ & $\begin{array}{l}\text { Group 2: } \\
\text { No } \\
\text { T2DM, } \\
\text { prior } \\
\text { CVD } \\
(n= \\
9,991)\end{array}$ & $\begin{array}{l}\text { Group 3: } \\
\text { T2DM, } \\
\text { no prior } \\
\text { CVD } \\
(n= \\
7,470)\end{array}$ & $\begin{array}{l}\text { Group } \\
\text { 4: } \\
\text { T2DM, } \\
\text { prior } \\
\text { CVD } \\
(n= \\
3,382)\end{array}$ & $p_{\text {value }}$ & $\begin{array}{l}\text { p value (for } \\
\text { comparison between } \\
\text { group } 2 \text { and } 3 \text { ) }\end{array}$ & $\begin{array}{l}\text { p value (for } \\
\text { comparison between } \\
\text { group } 3 \text { and } 4 \text { ) }\end{array}$ \\
\hline $\begin{array}{l}\text { All-cause } \\
\text { mortality, n } \\
(\%)\end{array}$ & $\begin{array}{l}9,183 \\
(27.8)\end{array}$ & $\begin{array}{l}3,385 \\
(33.9)\end{array}$ & $\begin{array}{l}2,318 \\
(31.0)\end{array}$ & $\begin{array}{l}1,360 \\
(40.2)\end{array}$ & $\begin{array}{l}< \\
0.001\end{array}$ & $<0.001$ & $<0.001$ \\
\hline $\begin{array}{l}\text { Non-fatal } \\
\text { AMI, n (\%) }\end{array}$ & $126(0.4)$ & $33(0.3)$ & $43(0.6)$ & $\begin{array}{l}26 \\
(0.8)\end{array}$ & 0.001 & 0.015 & 0.241 \\
\hline $\begin{array}{l}\text { Non-fatal } \\
\text { stroke, n } \\
(\%)\end{array}$ & 903 (2.7) & 321 (3.2) & $\begin{array}{l}228 \\
(3.1)\end{array}$ & $\begin{array}{l}132 \\
(3.9)\end{array}$ & $\begin{array}{l}< \\
0.001\end{array}$ & 0.547 & 0.022 \\
\hline $\begin{array}{l}\text { Heart } \\
\text { failure, } n \\
(\%)\end{array}$ & $\begin{array}{l}1,324 \\
(4.0)\end{array}$ & $534(5.3)$ & $\begin{array}{l}299 \\
(4.0)\end{array}$ & $\begin{array}{l}187 \\
(5.5)\end{array}$ & $\begin{array}{l}< \\
0.001\end{array}$ & $<0.001$ & $<0.001$ \\
\hline $\begin{array}{l}\text { CP01, n } \\
\text { (\%) }\end{array}$ & $\begin{array}{l}9,845 \\
(29.8)\end{array}$ & $\begin{array}{l}3,590 \\
(35.9)\end{array}$ & $\begin{array}{l}2,503 \\
(33.5)\end{array}$ & $\begin{array}{l}1,445 \\
(42.7)\end{array}$ & $\begin{array}{l}<.001 \\
0.001\end{array}$ & 0.001 & $<0.001$ \\
\hline $\begin{array}{l}\text { CPO2, n } \\
(\%)\end{array}$ & $\begin{array}{l}10,632 \\
(32.1)\end{array}$ & $\begin{array}{l}3,873 \\
(38.8)\end{array}$ & $\begin{array}{l}2,674 \\
(35.8)\end{array}$ & $\begin{array}{l}1,530 \\
(45.2)\end{array}$ & $\begin{array}{l}< \\
0.001\end{array}$ & $<0.001$ & $<0.001$ \\
\hline
\end{tabular}

AMI: Acute myocardial infarction. CP01: Composite primary outcome, number 1 (all-cause mortality, non-fatal myocardial infarction, non-fatal stroke). CPO2: Composite primary outcome, number 2 (all-cause mortality, non-fatal acute myocardial infarction, non-fatal stroke, heart failure).

Rates of all-cause mortality, CPO1 and CPO2 were higher in men than in women in every group (all p values $<0.05$ ), but the differences found for CPO1 and CPO2 were mainly driven by all-cause mortality (Supplemental Table S2). Table 3 shows the density incidence for all the outcomes in each population group. 
Table 3

Density incidence rates stratified by type 2 diabetes mellitus (T2DM) status, and presence or absence of prior cardiovascular disease (CVD).

\begin{tabular}{|c|c|c|c|c|}
\hline & $\begin{array}{l}\text { No T2DM, no prior } \\
\text { CVD }\end{array}$ & $\begin{array}{l}\text { No T2DM, prior } \\
\text { CVD }\end{array}$ & $\begin{array}{l}\text { T2DM, no prior } \\
\text { CVD }\end{array}$ & $\begin{array}{l}\text { T2DM, prior } \\
\text { CVD }\end{array}$ \\
\hline P-Y & $62,147.3$ & $18,107.7$ & $13,663.2$ & $5,892.8$ \\
\hline \multirow[t]{2}{*}{ Mortality rate per 1,000 P-Y (95\% Cl) } & 147.76 & 186.94 & 169.65 & 230.79 \\
\hline & $(144.74-150.78)$ & $(180.64-193.23)$ & $(162.75-176.56)$ & $\begin{array}{l}(218.53- \\
243.06)\end{array}$ \\
\hline \multirow{2}{*}{$\begin{array}{l}\text { Non-fatal AMI rate per 1,000 P-Y (95\% } \\
\mathrm{Cl})\end{array}$} & 2.03 & 1.82 & 3.15 & 4.41 \\
\hline & $(1.67-2.38)$ & $(1.20-2.44)$ & $(2.21-4.09)$ & $(2.72-6.11)$ \\
\hline \multirow{2}{*}{$\begin{array}{l}\text { Non-fatal stroke rate per } 1,000 \mathrm{P}-\mathrm{Y} \\
(95 \% \mathrm{Cl})\end{array}$} & 14.53 & 17.73 & 16.69 & 22.40 \\
\hline & $(13.58-15.48)$ & $(15.79-19.67)$ & $(14.52-18.85)$ & $(18.58-26.22)$ \\
\hline \multirow{2}{*}{$\begin{array}{l}\text { Heart failure rate per } 1,000 \text { P-Y }(95 \% \\
\mathrm{Cl})\end{array}$} & 21.30 & 29.49 & 21.88 & 31.73 \\
\hline & $(20.16-22.45)$ & $(26.99-31.99)$ & $(19.40-24.36)$ & $(27.19-36.28)$ \\
\hline \multirow[t]{2}{*}{ CP01 rate per 1,000 P-Y (95\% Cl) } & 158.41 & 198.26 & 183.19 & 245.22 \\
\hline & $(155.28-161.54)$ & $(191.77-204.74)$ & $(176.02-190.37)$ & $\begin{array}{l}(232.57- \\
257.86)\end{array}$ \\
\hline \multirow[t]{2}{*}{ CPO2 rate per 1,000 P-Y $(95 \% \mathrm{Cl})$} & 171.08 & 213.89 & 195.71 & 259.64 \\
\hline & $(167.83-174.33)$ & $(207.15-220.62)$ & $(188.29-203.13)$ & $\begin{array}{l}(246.63- \\
272.65)\end{array}$ \\
\hline
\end{tabular}

After adjusting for age, sex, age, sex, chronic obstructive pulmonary disease, solid cancer, leukemia/lymphoma, chronic kidney disease, dementia, heart failure, deep vein thrombosis/pulmonary thromboembolism, and atrial fibrillation, the presence of both prevalent T2DM plus established CVD (group 4) independently predicted all-cause mortality $(H R=1.48(95 \%$ $\mathrm{Cl}, 1.40$ to 1.57), as compared to the absence of both T2DM and established CVD (reference group 1) ( $p$ value < 0.01). CVD alone (group 2) and T2DM alone (group 3) also predicted all-cause mortality, showing similar HRs (1.13 and 1.14, respectively), which were lower than the HR that we are reporting for group 4 (Table 4). 
Table 4

Adjusted effect on all-cause mortality of the different categories according to type 2 diabetes mellitus (T2DM) status, and presence or absence of prior cardiovascular disease.

\begin{tabular}{|lllc|}
\hline & OR & Cl 95\% & P value \\
\hline Age & 1.10 & $1.09-1.11$ & $<0.01$ \\
\hline Male Gender & 1.23 & $1.19-1.28$ & $<0.01$ \\
\hline Group 1: T2DM (-) \& CVD (-) & 1 & & $<0.01$ \\
\hline Group 2: T2DM (-) \& CVD (+) & 1.13 & $1.09-1.18$ & $<0.01$ \\
\hline Group 3: T2DM (+) \& CVD (-) & 1.14 & $1.09-1.20$ & $<0.01$ \\
\hline Group 4: T2DM (+) \& CVD (+) & 1.48 & $1.40-1.57$ & \\
\hline $\begin{array}{l}\text { Adjusted by history of Chronic obstructive pulmonary disease, solid cancer, leukemia/lymphoma, chronic kidney disease, } \\
\text { dementia, heart failure, deep vein thrombosis or pulmonary thromboembolism, and atrial fibrillation. }\end{array}$ \\
\hline
\end{tabular}

\section{Discussion}

Here we observed significantly higher rates of cumulative incidence of all-cause mortality in participants with T2DM and established CVD. At the time of follow-up, participants with established CVD without T2DM presented higher rates of allcause mortality and heart failure than people with T2DM with no established CVD, albeit a lower rate of non-fatal acute myocardial infarction. Men significantly presented higher rates of all-cause mortality in each group, but we found no gender differences for incident non-fatal stroke or non-fatal myocardial infarction. In the model adjusted for age, sex, and the remaining clinical diagnoses included in the Charlson's comorbidity index, prevalent T2DM plus established CVD (group 4) independently predicted all-cause mortality as compared to the absence of both T2DM and established CVD, with a higher HR than either T2DM or established CVD taken separately.

When specifically analyzing cardiovascular disease in the very elderly, several distinctive characteristics of this population must be taken into consideration: first, there may be differences in the prevalence of traditional risk factors when compared with younger populations [17]; second, the impact of traditional cardiovascular risk factors on cardiovascular outcomes might decline with aging, whereas the importance of other factors such as frailty or inflammatory conditions as predisposing conditions to develop cardiovascular events may rise [18]; and third, the clinical approach to the very elderly may differ according to cultural or social disparities among different countries, for instance in the rate of deprescribing medications in the frail or highly dependent aged people [19].

It did not come as a surprise that in our study having both T2DM and established CVD conferred the highest risk of suffering cardiovascular events and all-cause death during follow-up. All-cause mortality risk was lower when either T2DM or established CVD were present in the absence of the other condition. Established cardiovascular disease increases the risk of repeated cardiovascular events and prevalent T2DM further increases that risk [20]. The VALIANT trial showed that both previously known diabetes and incident diabetes increased the risk of new cardiovascular events and death after an acute myocardial infarction [21]. Other authors have reported an even higher impact on behalf of T2DM on the prognoses of the elderly patients after a myocardial infarction than in younger patients [22]. Moreover, the increased mortality risk conferred by T2DM has also been described for stroke [23]. From a practical standpoint, patients who have had a cardiovascular event are categorized as having the highest cardiovascular risk, but this risk seems to be even higher if T2DM is present, both in the short and the long-term after the cardiovascular event $[24,25]$.

We noticed that nonagenarians with established CVD lacking the diagnosis of T2DM presented higher crude rates of allcause mortality than people with T2DM without established CVD. There has been a long-standing debate in the medical literature about whether prevalent T2DM and established coronary heart disease could be considered as "risk equivalent" for 
the development of cardiovascular events: some authors have defended this notion [26, 27], whilst others have not [28]. Additional research work brings diabetes at the same risk level as established cardiovascular disease but only for people with severe diabetes [29], or just for those with early-onset diabetes [30]. In the elderly, comorbidity and specific social and medical needs may make the comparison between both conditions to predict incident cardiovascular disease or mortality even less clear, since traditional risk factors lose weight at the same time that other conditions emerge to influence cardiovascular risk $[31,32]$. As a consequence, specific cardiovascular risk prediction tools for the elderly have been put in place $[33,34]$. Nevertheless, additional previous research has supported the idea of comparable cardiovascular risk between T2DM and established cardiovascular disease in the elderly [35]. In our study we found a similar all-cause mortality risk for both entities separately when we accounted for potential confounding variables.

We found no gender differences for incident non-fatal stroke or acute myocardial infarction, whereas all-cause mortality rate over time was higher in men. In a report from the TECOS study, even though women had worse cardiovascular risk factor profiles and were less often treated with indicated medications, they presented lower rates of cardiovascular events, which suggested that the cardioprotective effects of female sex extended to populations with T2DM [36]. On the opposite direction, other studies have found that the excess risk of acute coronary syndrome associated with T2DM is higher in women than in men [37]. Recent research in the Northern European population points to higher absolute rates of cardiovascular complications in men, whereas the relative rates of diabetes-related cardiovascular complications are higher in women than in men [38]. However, none of these studies focused on the very elderly, and methodological differences and distinct ethnic backgrounds may underlie the apparently discordant results.

We should point out some limitations of our study: some data were not available for a number of patients due to the fact that it was carried out under real conditions of clinical care. Thus, a possible bias due to missing data cannot completely be ruled out. However, recruitment bias was not an issue in our study, since we included virtually all the nonagenarians of our region. Also, we had no access to the cause of death of the participants; therefore, we designed modified variables for the combined endpoint "major cardiovascular events and mortality" -in our study, all-cause mortality instead of cardiovascular mortality was the outcome of interest-.

\section{Conclusions}

We found that prevalent T2DM plus established CVD independently predicted all-cause mortality as compared to the absence of both T2DM and established CVD. Established CVD and T2DM alone conferred similar mortality risks when they were present separately, which were of lower magnitude than the observed risk when both conditions were present. People with established CVD without T2DM presented higher rates of all-cause mortality and heart failure than people with T2DM without established CVD, but a lower rate of non-fatal acute myocardial infarction. Men significantly presented higher rates of allcause mortality in each group, but we found no gender differences for incident non-fatal stroke or non-fatal acute myocardial infarction. Additional research is needed to fully understand the impact of cardiovascular risk factors and the convenient approach to their clinical management in the very elderly.

\section{Abbreviations}

- Type 2 diabetes mellitus (T2DM)

- Cardiovascular disease (CVD)

- Chronic obstructive pulmonary disease (COPD)

- Chronic kidney disease (CKD)

- First composite primary outcome (CPO1) 
- Second composite primary outcome (CPO2)

- Standard deviation (SD)

- Analysis of variance (ANOVA)

- Confidence interval (Cl)

\section{Declarations}

- Ethics approval and consent to participate. Given that this study is retrospective and contains de-identified information, informed consent from the participants was waived by the Research Ethics Committee of the Ramon y Cajal University Hospital in Madrid, Spain (approval identification code: 113/16) on 22 April 2016. Also, our study was approved by the Research Ethics Committee of the La Paz University Hospital (approval identification code: PI-4296) on 27 July 2020, to study mortality rates after 30 months of follow-up. Lastly, an administrative permission to access electronic clinical records was acquired from the Research Central Commission of Madrid Regional Health Service (SERMAS), Primary Health Care (Madrid, Spain). The study complied with the International Guidelines for Ethical Review of Epidemiological Studies (Geneva, 1991).

- Consent for publication. Not applicable.

- Availability of data and materials. The datasets used and/or analysed during the current study are available from the corresponding author on reasonable request.

- Competing interests. The authors declare that they have no competing interests.

- Funding. This study has been funded by Instituto de Salud Carlos III through the projects "PI15/00259" and "PI18/01025", and co-funded by a European Regional Development Fund, "A way of shaping Europe". Also, was partially funded with assistance of the "Fundación para el Fomento y Desarrollo de la Investigación Clínica".

- Authors' contributions. MASF, contributed to the study design or concept and secured funds. JMP, CLR, JCV and JIVD performed the statistical analyses and edited the manuscript. JMDMY, PGC and MASF drafted the initial manuscript. All authors read and approved the final manuscript. All the authors met the criteria for authorship.

- Acknowledgements. This study was possible thanks to the physicians and nurses of the Madrid Primary Care Health Service, who provide optimal care to patients with chronic diseases.

- Patient and Public Involvement. Patients and/or the public were not involved in the design, or conduct, or reporting, or dissemination plans of this research.

\section{References}

1. Emerging Risk Factors Collaboration, Sarwar N, Gao P, Seshasai SR, Gobin R, Kaptoge S, Di Angelantonio E, Ingelsson E, Lawlor DA, Selvin E, Stampfer M, Stehouwer CD, Lewington S, Pennells L, Thompson A, Sattar N, White IR, Ray KK, Danesh J. Diabetes mellitus, fasting blood glucose concentration, and risk of vascular disease: a collaborative metaanalysis of 102 prospective studies. Lancet. 2010; 375:2215-22. doi: 10.1016/S0140-6736(10)60484-9.

2. Rao Kondapally Seshasai S, Kaptoge S, Thompson A, Di Angelantonio E, Gao P, Sarwar N, Whincup PH, Mukamal KJ, Gillum RF, Holme I, Njølstad I, Fletcher A, Nilsson P, Lewington S, Collins R, Gudnason V, Thompson SG, Sattar N, Selvin E, Hu FB, Danesh J; Emerging Risk Factors Collaboration. Diabetes mellitus, fasting glucose, and risk of cause-specific death. N Engl J Med. 2011; 364:829-41. doi: 10.1056/NEJMoa1008862.

3. García-García C, Subirana I, Sala J, Bruguera J, Sanz G, Valle V, Arós F, Fiol M, Molina L, Serra J, Marrugat J, Elosua R. Long-term prognosis of first myocardial infarction according to the electrocardiographic pattern (ST elevation 
myocardial infarction, non-ST elevation myocardial infarction and non-classified myocardial infarction) and revascularization procedures. Am J Cardiol. 2011; 108:1061-7. doi: 10.1016/j.amjcard.2011.06.003.

4. Kolominsky-Rabas PL, Weber M, Gefeller O, Neundoerfer B, Heuschmann PU. Epidemiology of ischemic stroke subtypes according to TOAST criteria: incidence, recurrence, and long-term survival in ischemic stroke subtypes: a populationbased study. Stroke. 2001; 32:2735-40.

5. Becker A, Bos G, de Vegt F, Kostense PJ, Dekker JM, Nijpels G, Heine RJ, Bouter LM, Stehouwer CD. Cardiovascular events in type 2 diabetes: comparison with nondiabetic individuals without and with prior cardiovascular disease. 10-year follow-up of the Hoorn Study. Eur Heart J. 2003; 24:1406-13.

6. Muñoz-Rivas N, Méndez-Bailón M, Hernández-Barrera V, de Miguel-Yanes JM, Jiménez-García R, Esteban-Hernández J, Jiménez-Trujillo I, Alvaro-Meca A, Carrasco-Garrido P, de Miguel-Díez J, López-de-Andrés A. Time trends in ischemic stroke among type 2 diabetic and non-diabetic patients: Analysis of the Spanish National Hospital Discharge data (2003-2012). PLoS One. 2015; 10:e0145535. doi: 10.1371/journal.pone.0145535. eCollection 2015.

7. Rich MW, Chyun DA, Skolnick AH, Alexander KP, Forman DE, Kitzman DW, Maurer MS, McClurken JB, Resnick BM, Shen WK, Tirschwell DL; American Heart Association Older Populations Committee of the Council on Clinical Cardiology, Council on Cardiovascular and Stroke Nursing, Council on Cardiovascular Surgery and Anesthesia, and Stroke Council; American College of Cardiology; and American Geriatrics Society. Knowledge gaps in cardiovascular care of the older adult population: A scientific statement from the American Heart Association, American College of Cardiology, and American Geriatrics Society. Circulation. 2016; 133:2103-22. doi: 10.1161/CIR.0000000000000380.

8. Zulman DM, Sussman JB, Chen X, Cigolle CT, Blaum CS, Hayward RA Examining the evidence: a systematic review of the inclusion and analysis of older adults in randomized controlled trials. J Gen Intern Med. 2011;26:783-90. doi: 10.1007/s11606-010-1629-x.

9. American Diabetes Association. 12. Older Adults: Standards of Medical Care in Diabetes-2021. Diabetes Care. 2021 Jan;44(Suppl 1):S168-S179. doi: 10.2337/dc21-S012.

10. Beckett NS, Peters R, Fletcher AE, Staessen JA, Liu L, Dumitrascu D, Stoyanovsky V, Antikainen RL, Nikitin Y, Anderson C, Belhani A, Forette F, Rajkumar C, Thijs L, Banya W, Bulpitt CJ; HYVET Study Group. Treatment of hypertension in patients 80 years of age or older. N Engl J Med. 2008; 358:1887-98. doi: 10.1056/NEJMoa0801369.

11. Salinero-Fort MA, Mostaza-Prieto JM, Lahoz-Rallo C, Vicente Díez JI, Cárdenas-Valladolid J. Population-based crosssectional study of 11645 Spanish nonagenarians with type 2 diabetes mellitus: cardiovascular profile, cardiovascular preventive therapies, achievement goals and sex differences. BMJ Open. 2019;9(9):e030344. doi: 10.1136/bmjopen2019-030344.

12. Cabañero-Martínez MJ, Cabrero-García J, Richart-Martínez M, Muñoz-Mendoza CL. The Spanish versions of the Barthel index (BI) and the Katz index (KI) of activities of daily living (ADL): a structured review. Arch Gerontol Geriatr. 2009; 49:e77-84. doi: 10.1016/j.archger.2008.09.006.

13. Vergara I, Bilbao A, Orive M, Garcia-Gutierrez S, Navarro G, Quintana JM. Validation of the Spanish version of the Lawton IADL Scale for its application in elderly people. Health Qual Life Outcomes. 2012; 10:130. doi: 10.1186/1477-7525-10130.

14. Charlson ME, Pompei P, Ales KL, MacKenzie CR. A new method of classifying prognostic comorbidity in longitudinal studies: development and validation. J Chronic Dis. 1987; 40:373-83.

15. de Burgos-Lunar C, Salinero-Fort MA, Cárdenas-Valladolid J, Soto-Díaz S, Fuentes-Rodríguez CY, Abánades-Herranz JC, del Cura-González I. Validation of diabetes mellitus and hypertension diagnosis in computerized medical records in primary health care. BMC Med Res Methodol. 2011; 11:146. doi: 10.1186/1471-2288-11-146.

16. Gil Montalbán E, Ortiz Marrón H, López-Gay Lucio-Villegas D, Zorrilla Torrás B, Arrieta Blanco F, Nogales Aguado P. Validity and concordance of electronic health records in primary care (AP-Madrid) for surveillance of diabetes mellitus. PREDIMERC study. Gac Sanit. 2014; 28:393-6. doi: 10.1016/j.gaceta.2014.03.001. 
17. Wang R, Fratiglioni L, Liang Y, Welmer AK, Xu W, Mangialasche F, Johnell K, Qiu C. Prevalence, pharmacological treatment, and control of cardiometabolic risk factors among older people in central Stockholm: a population-based study. PLoS One. 2015; 10:e0119582. doi: 10.1371/journal.pone.0119582.

18. Lind L, Sundström J, Ärnlöv J, Lampa E. Impact of Aging on the Strength of Cardiovascular Risk Factors: A Longitudinal Study Over 40 Years. J Am Heart Assoc. 2018; 7. pii: e007061. doi: 10.1161/JAHA.117.007061.

19. Farrell B, Tsang C, Raman-Wilms L, Irving H, Conklin J, Pottie K. What Are Priorities for Deprescribing for Elderly Patients? Capturing the Voice of Practitioners: A Modified Delphi Process. PLoS One. 2015; 10:e0122246. doi: 10.1371/journal.pone.0122246.

20. Melchior T, Kober L, Madsen CR, Seibaek M, Jensen GV, Hildebrandt P, Torp-Pedersen C. Accelerating impact of diabetes mellitus on mortality in the years following an acute myocardial infarction. TRACE Study Group. Trandolapril Cardiac Evaluation. Eur Heart J. 1999; 20:973-8.

21. Aguilar D, Solomon SD, Køber L, Rouleau JL, Skali H, McMurray JJ, Francis GS, Henis M, O'Connor CM, Diaz R, Belenkov YN, Varshavsky S, Leimberger JD, Velazquez EJ, Califf RM, Pfeffer MA. Newly diagnosed and previously known diabetes mellitus and 1-year outcomes of acute myocardial infarction: the VALsartan In Acute myocardial iNfarcTion (VALIANT) trial. Circulation. 2004; 110:1572-8.

22. Afanasiev SA, Garganeeva AA, Kuzheleva EA, Andriyanova AV, Kondratieva DS, Popov SV. The impact of type 2 diabetes mellitus on long-term prognosis in patients of different ages with myocardial infarction. J Diabetes Res. 2018; 2018:1780683. doi: 10.1155/2018/1780683. Corrigendum in: J Diabetes Res. 2019; 2019:8347891. doi: $10.1155 / 2019 / 8347891$.

23. Liao CC, Shih CC, Yeh CC, Chang YC, Hu CJ, Lin JG, Chen TL. Impact of diabetes on stroke risk and outcomes: Two nationwide retrospective cohort studies. Medicine (Baltimore). 2015; 94:e2282. doi: 10.1097/MD.0000000000002282

24. Nauta ST, Deckers JW, Akkerhuis KM, van Domburg RT. Short- and long-term mortality after myocardial infarction in patients with and without diabetes: changes from 1985 to 2008. Diabetes Care. 2012; 35:2043-7.

25. Kümler T, Gislason GH, Køber L, Torp-Pedersen C. Diabetes is an independent predictor of survival 17 years after myocardial infarction: follow-up of the TRACE registry. Cardiovasc Diabetol. 2010; 9:22. doi: 10.1186/1475-2840-9-22.

26. Haffner SM, Lehto S, Rönnemaa T, Pyörälä K, Laakso M. Mortality from coronary heart disease in subjects with type 2 diabetes and in nondiabetic subjects with and without prior myocardial infarction. N Engl J Med. 1998; 339:229-34.

27. Juutilainen A, Lehto S, Rönnemaa T, Pyörälä K, Laakso M. Type 2 diabetes as a "coronary heart disease equivalent": an 18-year prospective population-based study in Finnish subjects. Diabetes Care. 2005; 28:2901-7.

28. Carnethon MR, Biggs ML, Barzilay J, Kuller LH, Mozaffarian D, Mukamal K, Smith NL, Siscovick D. Diabetes and coronary heart disease as risk factors for mortality in older adults. Am J Med. 2010; 123:556.e1-9. doi: 10.1016/j.amjmed.2009.11.023.

29. Mondesir FL, Brown TM, Muntner P, Durant RW, Carson AP, Safford MM, Levitan EB. Diabetes, diabetes severity, and coronary heart disease risk equivalence: REasons for Geographic and Racial Differences in Stroke (REGARDS). Am Heart J. 2016; 181:43-51. doi: 10.1016/j.ahj.2016.08.002.

30. Wannamethee SG, Shaper AG, Whincup PH, Lennon L, Sattar N. Impact of diabetes on cardiovascular disease risk and all-cause mortality in older men: influence of age at onset, diabetes duration, and established and novel risk factors. Arch Intern Med. 2011; 171:404-10. doi: 10.1001/archinternmed.2011.2.

31. van Bussel EF, Richard E, Busschers WB, Steyerberg EW, van Gool WA, Moll van Charante EP, Hoevenaar-Blom MP. A cardiovascular risk prediction model for older people: Development and validation in a primary care population. J Clin Hypertens (Greenwich). 2019; 21:1145-1152. doi: 10.1111/jch.1361.

32. Beer C, Alfonso H, Flicker L, Norman PE, Hankey GJ, Almeida OP. Traditional risk factors for incident cardiovascular events have limited importance in later life compared with the health in men study cardiovascular risk score. Stroke. 2011; 42:952-9. doi: 10.1161/STROKEAHA.110.603480.

Page 13/14 
33. Cooney MT, Selmer R, Lindman A, Tverdal A, Menotti A, Thomsen T, DeBacker G, De Bacquer D, Tell GS, Njolstad I, Graham IM; SCORE and CONOR investigators. Cardiovascular risk estimation in older persons: SCORE O.P. Eur J Prev Cardiol. 2016; 23:1093-103. doi: 10.1177/2047487315588390.

34. Simons LA, Simons J, Friedlander Y, McCallum J, Palaniappan L. Risk functions for prediction of cardiovascular disease in elderly Australians: the Dubbo Study. Med J Aust. 2003; 178:113-6.

35. Barzilay JI, Mukamal KJ, Kizer JR. Atherosclerotic cardiovascular disease in older adults with diabetes mellitus. Clin Geriatr Med. 2015; 31:29-39, vii. doi: 10.1016/j.cger.2014.09.001.

36. Alfredsson J, Green JB, Stevens SR, Reed SD, Armstrong PW, Angelyn Bethel M, Engel SS, McGuire DK, Van de Werf F, Hramiak I, White HD, Peterson ED, Holman RR; TECOS Study Group. Sex differences in management and outcomes of patients with type 2 diabetes and cardiovascular disease: A report from TECOS. Diabetes Obes Metab. 2018; 20:237988. doi: 10.1111/dom.13377.

37. Dong X, Cai R, Sun J, Huang R, Wang P, Sun H, Tian S, Wang S. Diabetes as a risk factor for acute coronary syndrome in women compared with men: a meta-analysis, including 10,856,279 individuals and 106,703 acute coronary syndrome events. Diabetes Metab Res Rev. 2017; 33. doi: 10.1002/dmrr.2887.

38. Malmborg M, Schmiegelow MDS, Nørgaard CH, Munch A, Gerds T, Schou M, Kistorp C, Torp-Pedersen C, Hlatky MA, Gislason G. Does type 2 diabetes confer higher relative rates of cardiovascular events in women compared with men? Eur Heart J. 2019. pii: ehz913. doi: 10.1093/eurheartj/ehz913.

\section{Supplementary Files}

This is a list of supplementary files associated with this preprint. Click to download.

- SupplementalTables1.docx

- SupplementalTables2.docx 